\title{
Incidence of histoplasmosis in a cohort of people with HIV: from estimations to reality
}

\author{
Narda Medina ${ }^{1,+}$, Juan Luis Rodriguez-Tudela ${ }^{2,+}$, Luis Aguirre ${ }^{1,+}{ }^{+}$, Luis R. Salazar ${ }^{1,+}$, Osmar Gamboa1, ${ }^{1,}$, Oscar Boni- \\ lla $^{3,+}$, Juan C. Pérez ${ }^{3,+}$, Eduardo Arathoon ${ }^{1,3,+}$, David W. Denning $2,4,5,+$, Ana Alastruey-Izquierdo ${ }^{2,6 *}$
}

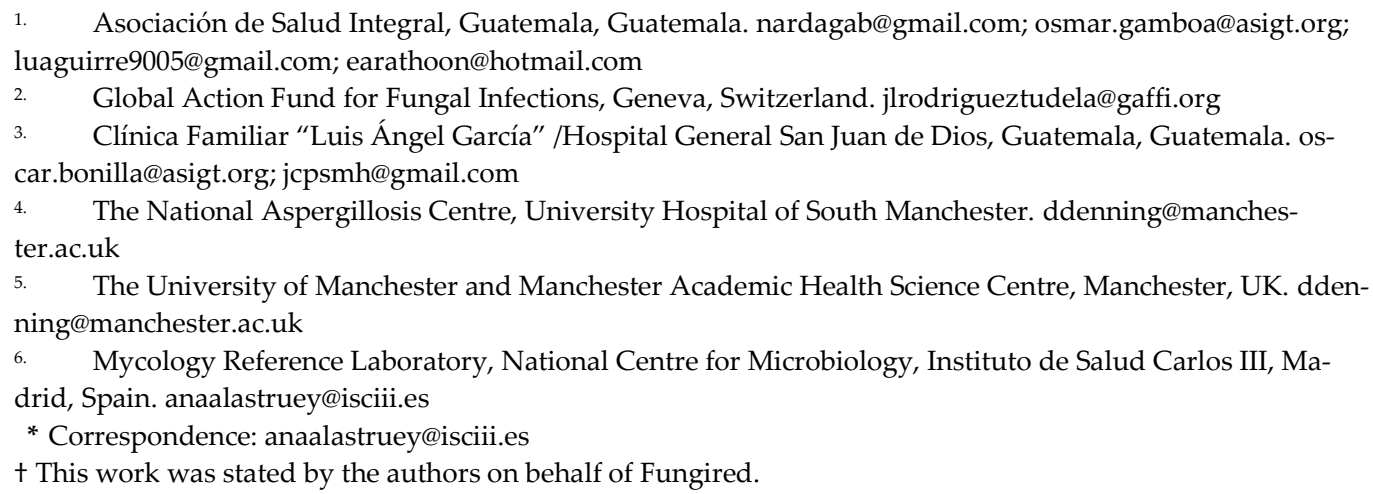

\begin{abstract}
Among people with HIV, histoplasmosis represents an important cause of mortality. Previous studies have provided estimates of the disease incidence. Here, we compared those estimates with the results obtained from a screening program implemented in Guatemala, which included histoplasmosis detection for people with HIV. To compare the results of this program, with previous estimations, a literature search was done and reports about histoplasmosis incidence were analyzed. The screening program enrolled 6,366 patients. The overall histoplasmosis incidence in the screening program was $7.4 \%$, which was almost double than those estimated by the previous studies. From 2017 to 2019 , the screening program showed an upward trend in histoplasmosis cases from $6.5 \%$ to $8.8 \%$. Histoplasmosis overall mortality among those who were newly HIV diagnosed showed a decrease at 180 days from $32.8 \%$ in 2017 to $21.2 \%$ in 2019 . The screening approach using rapid diagnostic assays detects quickly more cases of histoplasmosis, allowing a specific treatment, which decreases the mortality of the disease. Therefore, the use of these new techniques, especially in endemic areas of histoplasmosis, must be implemented.
\end{abstract}

Keywords: Histoplasmosis; Antigen; HIV; Opportunistic infections

\section{Introduction}

In recent years histoplasmosis has been recognized as an important opportunistic infection among people with HIV in Latin America [1,2]. However, until the development and commercialization of the antigen detection assay, the diagnosis was cumbersome. It involved invasive procedures, which were unavailable in many low- and middle-income countries [3,4]. Classical diagnostic procedures such as blood culture were the gold standard for diagnosis of histoplasmosis. However, they required complex laboratory infrastructure (Biosafety Level 3), are time-consuming, and have a limited sensitivity to detect the disease, from 36 to $77 \%$ [1,5]. In this context, accurate diagnosis was challenging. Recently, the antigen detection assay has shown a high analytical sensitivity and specificity with $98 \%$ and $97 \%$, respectively [1,6]. Therefore, access to this test is providing a better understanding of the real disease burden.

In Guatemala, since 2017, a screening program for people with HIV has provided, among other opportunistic infections, the detection of histoplasmosis. This program has 
allowed to quantify the real incidence of this disease in people with HIV. Here, we compare histoplasmosis incidence published in the literature and the results of the screening program implemented in Guatemala from 2017 to 2019, showing the implications that the different approaches have in the care of population with HIV.

\section{Materials and Methods}

\subsection{Study design and participants}

A literature search was done in July 2021 to identify published studies where the incidence of histoplasmosis in Guatemala was reported using MEDLINE, Scielo, and Google Scholar. Then we compared the absolute differences in histoplasmosis incidence between these analyses and data obtained from the screening program performed from 2017-2019. Detailed description of the program has been published previously [7,8]. Briefly, patients were enrolled at the health care facilities that are part of a national network that covers $81.2 \%$ (13 out of 16 ) of the sites that provide care to HIV-infected patients in the country. The program included three groups of HIV patients: i) newly diagnosed, ii) patients who are not receiving antiretrovirals treatment (ART) for $>90$ days but who returned to care (Return/Restart), and iii) patients on ART with symptoms of opportunistic infections (ARV treatment). The clinical samples for the screening were sent from the health care facilities to a central diagnostic laboratory hub located in Guatemala City. Patients were screened for tuberculosis (TB), nontuberculous mycobacteria (NTM), histoplasmosis and cryptococcal disease, independently of their CD4 cell count. A diagnosis of histoplasmosis was considered when the antigen detection, culture or PCR test was positive. Advanced HIV disease (AHD) was defined as having a CD4 count $<200 \mathrm{cells} / \mathrm{mm}^{3}$. In this analysis we describe histoplasmosis trends for 2017 to 2019 as well as the reported numbers of deaths at 180 days of follow-up.

Demographic data were collected in the health care facilities using a standard electronic form. Patients received ART and treatment for opportunistic infections in accordance with the national guidelines [9]. Histoplasmosis refers to a positive result for Isolator blood culture, urine antigen and/or a histoplasma PCR, not subacute disseminated or chronic pulmonary histoplasmosis, which have not been estimated or documented.

\subsection{Statistical analysis}

Differences between incidence obtained in the literature and this study were compared in absolute numbers. For baseline characteristics of the histoplasmosis and trends, a descriptive statistical analysis was performed using SPSS calculating proportions. Graphics were developed in Microsoft Excel. Incidences were calculated from the patients tested globally and for each group of patients using percentages. Histoplasmosis-related mortality was analyzed with Kaplan-Meier at 180 days of follow-up.

\section{Results}

Two previous publications reported histoplasmosis incidence among people with HIV in Guatemala. In 2018, Adenis et al. estimated an overall histoplasmosis incidence of $1.48 \%$ for the Latin American region, with Guatemala showing a $4.16 \%$. This accounted for 2,676 cases [10]. Previously, in 2015, Medina et al. had estimated the incidence in 3.8\% [11]. However, case incidence of $7.4 \%$ in the screening program is about two-fold or an additional 1,523 cases. A total of 6,366 patients were included in the screening program. The overall incidence per year found was $6.5 \%$ in $2017,6.8 \%$ in 2018 , and $8.8 \%$ in 2019 showing a statistically significant upward trend in the absolute number of histoplasmosis cases $(P=0.005)$. However, there were differences among the three group of patients. Thus, newly diagnosed HIV patients had a histoplasmosis incidence of $8.5 \%$, patients who return to care, $8.3 \%$, and those on ART, $4.8 \%$ (Figure 1). We observed a steady annual increase in histoplasmosis incidence within the newly diagnosed HIV patients and patients 
on ARV while the other group, patients who return to care, showed a decrease in 2018. Table 1 shows the annual incident histoplasmosis cases per type of patients.

At enrollment, CD4 cell counts were available for 4,625 patients $(72.5 \%)$; of those, $2,235(48.3 \%)$ had AHD. The incidence of histoplasmosis in AHD was $11.9 \%$ among the newly diagnosed HIV patients in comparison with $2.7 \%$ in those without AHD. In the group of patients on antiretroviral therapy and those who returned to care with AHD the histoplasmosis incidence was $8.6 \%$ and $11.5 \%$, respectively.

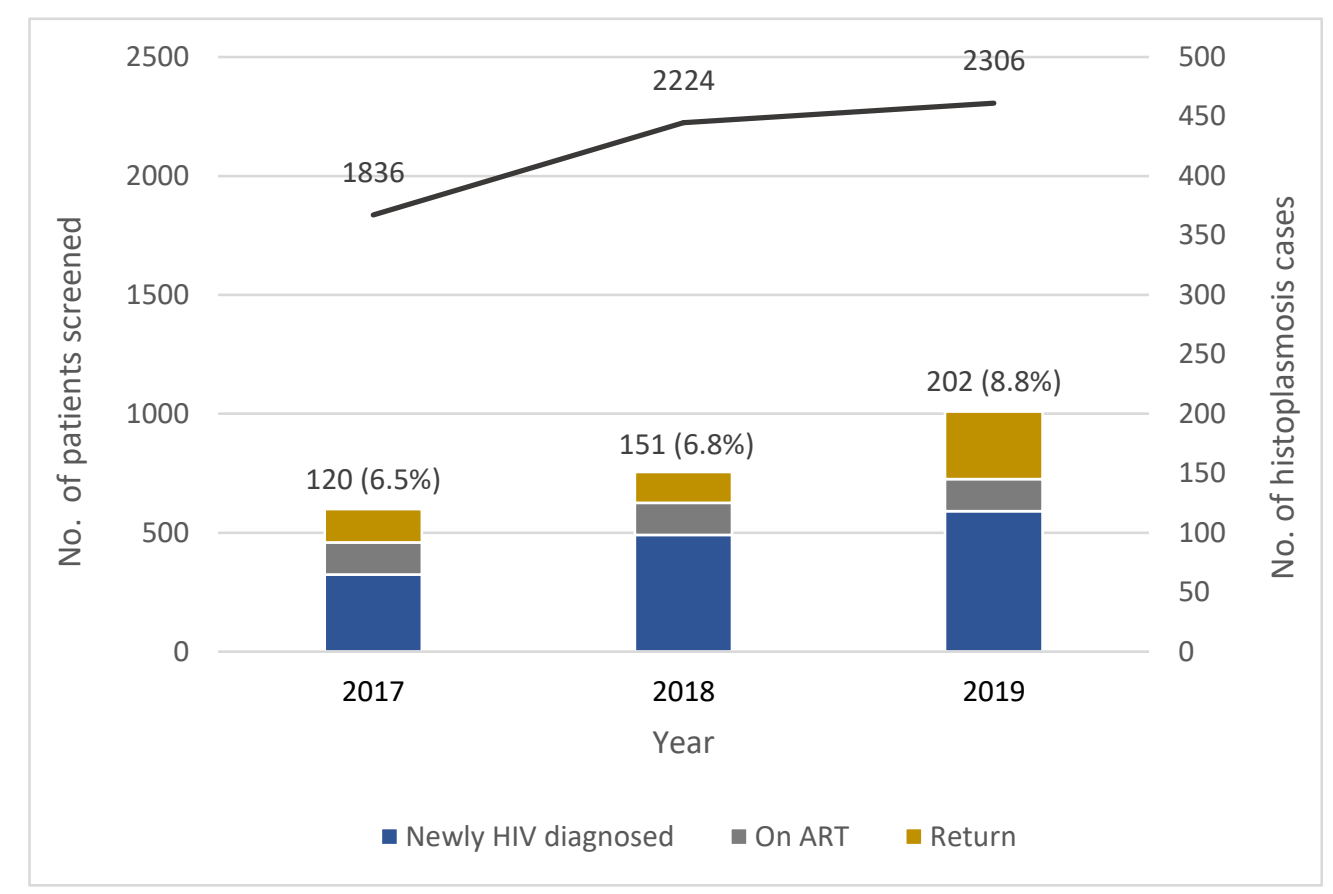

Figure 1. Annual screening patient numbers and histoplasmosis cases detected in the OI program

Table 1. Incidence of histoplasmosis among different groups of patients, by year

\begin{tabular}{cccccc}
\hline \multirow{2}{*}{ Type of patient } & $\begin{array}{c}\text { Screened } \\
\text { patients }\end{array}$ & $\begin{array}{c}\text { Overall } \\
\text { incidence }\end{array}$ & $\begin{array}{c}\text { Year } \\
\mathbf{2 0 1 7}\end{array}$ & $\begin{array}{c}\text { Year } \\
\mathbf{2 0 1 8}\end{array}$ & $\begin{array}{c}\text { Year } \\
\mathbf{2 0 1 9}\end{array}$ \\
\hline Newly diagnosed HIV & 3322 & $8.5 \%$ & $7.5 \%$ & $8.3 \%$ & $9.3 \%$ \\
On ART & 1704 & $4.8 \%$ & $4.1 \%$ & $4.6 \%$ & $5.9 \%$ \\
Return to care & 1338 & $8.3 \%$ & $9.2 \%$ & $5.8 \%$ & $9.8 \%$ \\
Overall & 6366 & $7.4 \%$ & $6.5 \%$ & $6.8 \%$ & $8.8 \%$ \\
\hline
\end{tabular}

ART = antiretroviral therapy

Among those who had histoplasmosis, most patients were male (65.8\%) between 28 to 42 years old. Sexual orientation, and area of residence were found to be associated with histoplasmosis status through chi-squared analysis $(P<0.005)$. Histoplasmosis incidence in rural areas was higher than in urban areas $(9.0 \%$ vs $4.9 \%, P<0.0001)$ as well as among heterosexual men vs men who have sex with men $(8.1 \%$ vs $4.9 \%, P<0.0001)$. Coinfection was found in 74 (15.6\%) out of 473 patients diagnosed with histoplasmosis. The most common dual or triple infection was histoplasmosis plus tuberculosis with 42 cases (56.8\%), followed by histoplasmosis plus cryptococcosis, 22 cases $(29.7 \%)$, histoplasmosis plus NTM, 7 cases (9.5\%) and histoplasmosis-cryptococcosis-tuberculosis, 3 cases (4.1\%). Pneumocystis pneumonia and esophageal candidosis data were not systematically collected during these years. Table 2 summarizes several characteristics of the screened patients. 
Four hundred and sixty-nine patients with histoplasmosis were followed up at 180 days; of whom 131 deaths (27.9\%) were reported. Eighty-seven (66.4\%) of these deaths occurred in the first 30 days. Table 3 shows histoplasma mortality at 180 days per type of patient and year. Among newly diagnosed HIV patients there was a decline of histoplasmosis deaths along with the overall decline of mortality (32.8\% in 2017 to $21.6 \%$ in 2019; $P=0.0626$ ). However, patients who returned to care and those on ARV showed a mortality increase at 180 days, in 2018 and decline in 2019. The number of patients receiving histoplasmosis treatment in those newly diagnosed with HIV increased from $84.6 \%$ in 2017 to $92.4 \%$ in 2019 , whereas in those who returned to care increased slightly from $82.1 \%$ in 2017 to $86 \%$ in 2019 , and in patients on ART from $74.1 \%$ in 2017 to $85.2 \%$ in 2019 . Amphotericin B deoxycholate and itraconazole were the drugs available in the country during the study period.

Table 2. Baseline characteristics of patients screened for histoplasmosis

\begin{tabular}{|c|c|c|c|c|c|}
\hline \multirow[t]{2}{*}{ Characteristics } & \multirow[t]{2}{*}{$\begin{array}{l}\text { Number of patients } \\
\text { screened }\end{array}$} & \multicolumn{2}{|c|}{$\begin{array}{l}\text { Histoplasmosis } \\
\qquad(\mathrm{n}=473)\end{array}$} & \multicolumn{2}{|c|}{$\begin{array}{c}\text { Non-histoplasmosis } \\
(n=5893)\end{array}$} \\
\hline & & $\mathbf{n}$ & $\%$ & $\mathbf{n}$ & $\%$ \\
\hline \multicolumn{6}{|l|}{ Sex } \\
\hline Male & 4049 & 311 & $65.8 \%$ & 3738 & $63.4 \%$ \\
\hline Female & 2252 & 158 & $33.4 \%$ & 2094 & $35.5 \%$ \\
\hline Transsexual & 65 & 4 & $0.8 \%$ & 61 & $1.0 \%$ \\
\hline Age (years), median (IQR) & 6360 & 35 & $(28-42)$ & 34 & $(27-43)$ \\
\hline \multicolumn{6}{|l|}{ Sexual orientation } \\
\hline Heterosexual & 4796 & 389 & $82.2 \%$ & 4407 & $74.8 \%$ \\
\hline Homosexual & 1083 & 53 & $11.2 \%$ & 1030 & $17.5 \%$ \\
\hline Bisexual & 371 & 19 & $4.0 \%$ & 352 & $6.0 \%$ \\
\hline Unknown & 116 & 12 & $2.5 \%$ & 104 & $1.8 \%$ \\
\hline \multicolumn{6}{|l|}{ Residence } \\
\hline Urban & 3015 & 163 & $35.2 \%$ & 2852 & $49.8 \%$ \\
\hline Rural & 3180 & 300 & $64.8 \%$ & 2880 & $50.2 \%$ \\
\hline \multicolumn{6}{|l|}{ Ethnic group } \\
\hline Ladino & 4909 & 368 & $77.8 \%$ & 4541 & $77.1 \%$ \\
\hline Mayan & 914 & 67 & $14.2 \%$ & 847 & $14.4 \%$ \\
\hline Other & 45 & 0 & $0 \%$ & 45 & $0.8 \%$ \\
\hline Unknown & 498 & 38 & $8.0 \%$ & 460 & $7.8 \%$ \\
\hline
\end{tabular}

Table 3. Histoplasma mortality at 180 days of follow-up among HIV patients

\begin{tabular}{ccccc}
\hline \multirow{2}{*}{ Type of patient } & Overall & \multicolumn{3}{c}{ Mortality by year } \\
\cline { 3 - 5 } mortality & $\mathbf{2 0 1 7}$ & $\mathbf{2 0 1 8}$ & $\mathbf{2 0 1 9}$ \\
\hline Newly diagnosed HIV & 27.6 & 32.8 & 32.0 & 21.2 \\
On ART & 30.0 & 30.8 & 40.7 & 18.5 \\
Return to care & 27.3 & 21.4 & 30.8 & 28.6 \\
\hline
\end{tabular}

\section{Discussion}

Fungal diseases are generally neglected for many reasons. Diagnosis is often complicated, although new detection methods have become available. Furthermore, the content of education programs about fungal diseases is a minor topic in medical schools and even 
in continuous education postgraduate programs. This means that clinical suspicion of fungal disease is limited even though many of these infections are life threatening. This low level of suspicion implies that clinicians do not request appropriate diagnosis and consequently many laboratories do not have the expertise and techniques required for prompt detection or exclusion of serious fungal disease. The project performed in Guatemala illustrates this situation. Using a screening strategy in high-risk patients with HIV infection, irrespective of the clinical suspicion and immunological status, we have found that histoplasmosis has been routinely underestimated. Our program provides much more accurate incidence data, allowing the allocation of the correct tools to diagnose and treat this disease in the country. Guatemala has limited resources, but appropriate rapid screening for histoplasmosis is clearly worthwhile in HIV patients.

The use of a central diagnostic laboratory hub for the screening program versus local laboratories should be analyzed carefully. A centralized laboratory, such as that implemented in Guatemala offers advantages such as lower cost per sample, more expertise, easy quality control of the test, and centralized epidemiological data. On the other hand, local laboratories are closer to the patient which should be able to make the results available even more quickly. However, it is important to consider the number of samples that local laboratories run per week and the availability of a quality control program to warrant the right performance of the whole diagnostic process. Successful national or regional implementation through a central laboratory must provide a good system for delivering samples from the peripherical centers linked with a structured program to facilitate point of care tests (when they are available to use) at the bedside or in clinic.

Over the study period, we observed a general upward trend in histoplasmosis detection. In 2012, the national epidemiological report identified only 32 cases of histoplasmosis in the country [11]. In the screening program a diagnosis of histoplasmosis was reached in 158 people on average per year, among more than 1,800 tests performed. Access to diagnosis has been the key change in the care of people with HIV in Guatemala, allowing more accurate epidemiological data and better clinical care. A key component of the project has been a training program. Unexpected diagnostic results of some tests have helped clinicians to be more aware of histoplasmosis, request more tests and treat patients quickly and appropriately, both for histoplasmosis alone and in cases with dual or triple infections. The increasing histoplasmosis incidence shown in this analysis should spur others to make more efforts to reach all cases at risk with rapid diagnostic testing.

Comparing our results with the previously published data, the introduction of the new assays such as the antigen Histoplasma detection test is mandatory. This assay is affordable, provides rapid results, and the clinical sample tested is urine, which is easily collected. Literature data shows an underestimation of histoplasmosis cases that can be related to the use of tests with low sensitivity, the nature of the studies, and lack of clinical suspicion. Histoplasmosis incidence in the screening program was about two-fold higher than the previous estimate by Adenis et al. (7.4\% vs 4.16\%) [12]. Guatemala is known as a hyperendemic country with one of the highest incidences of histoplasmosis in Latin America. Our study shows an excess of 1,523 cases of histoplasmosis among people with HIV in Guatemala, over prior estimates. Other countries such as Guyana (2.76\%), Venezuela (2.90\%), Argentina (1.89\%), and the Central America region were also cataloged with high incidence levels $(\geq 1.5 \%)$ [10], but the annual incidence is probably underestimated too. Unavailability of laboratory tests and lack of screening strategies in patients at high risk of opportunistic infections are related to underestimation of the burden of diseases. Moreover, in people with HIV, histoplasmosis symptoms are nonspecific and are often indistinguishable from other diseases like tuberculosis. Examples of common clinical features which can be confused with other disease entities include non-specific respiratory complains and pulmonary infiltrates (TB, Pneumocystis or bacterial pneumonia), pancytopenia (HIV, visceral leishmaniasis, lymphoma, drug toxicity), hepatosplenomegaly (leishmaniasis, lymphoma, disseminated TB), diarrhea and weight loss (HIV, disseminated NTM infection, intestinal infection) and others. As our work shows, histoplasmosis 
can be present with other HIV complication in the same patient. Frequencies of this cooccurrence have been reported from 9 to $38 \%$ [13-15].

We have also found that the incidence of histoplasmosis is almost double in rural than in urban areas of Guatemala. Many studies are conducted in urban settings only. In 1960, a histoplasmin skin survey was performed showing an overall reactivity in the general population of $57.2 \%$ [16] with the rural areas having the highest frequencies in accord with our current findings. According to the national HIV continuum report, facilities included in the screening program provide health care attention to approximately $60 \%$ of population with HIV in the country [17]. Therefore, our epidemiology data is a robust estimate and indicates that diagnostic services need to reach rural areas for histoplasmosis.

The screening program provided access to diagnosis independently of the CD4 cell count. If a limit of $<200$ cells $/ \mathrm{mm}^{3}$ had been used, $27.5 \%$ of the patients would not have been screened. In our study, $92 \%$ of the histoplasmosis cases occurred in patients with $<350$ CD4 cells. Our findings also showed that the overall histoplasmosis mortality was $29.6 \%$ in $2017,33.3 \%$ in 2018 , and $22.8 \%$ in 2019 . Despite the 2018 , there was an overall reduction in mortality from 2017 to 2019 of $6.8 \%$. The reduction in histoplasmosis mortality was observed in those with newly diagnosed HIV from $32.8 \%$ in 2017 to $21.2 \%$ in 2019. Clearly, the access to diagnosis for histoplasmosis is essential to guide right treatment decisions. Patients who return to care and those on ART showed an unexpectedly increased mortality for histoplasmosis in 2018. Although there is no clear explanation for this, the unavailability of rapid results of the immunological and virological status of patients already entered in ARV program could have influenced the speed of diagnosis requests and treatment approach.

This study has some limitations. First, differences between health care facilities in the way they actively search for patients to return to treatment and on the way patients on ARV are managed might have influenced the screened group. Second, we have not collected patient's clinical information, therefore we were not able to determine the number of incident cases that presented with and without symptoms, and which clinical presentation. Despite these limitations the program has gathered a significant amount of data due to the active screening providing a new perspective for the estimations of burden of disease as well as allowing access to diagnosis for life-threatening infections in a high-risk population of patients. Therefore, we strongly recommend a screening approach in high burden locations and countries for several reasons: (i) the high rate of coinfections in this population; (ii) the difficulties in distinguishing tuberculosis and other conditions, and (iii) as an indirect tool for continuing education of clinicians which forces them to make a careful evaluation of the patient to confirm an unexpected laboratory diagnosis as well as to take treatment decisions to save patients' lives. There is a clear need to reinforce diagnostic laboratories in low- and middle-income countries. The new diagnostic tools with a high sensitivity and specificity have changed the landscape of diagnosis of fungal infections and can be implemented very quickly. Training of clinicians is also required alongside the introduction of new tests. This can take a long time if the disease is unfamiliar. By introducing screening, we can quickly improve the quality of patient's care as it requires a clinical response when tests are positive. Strategic introduction of Diagnostic Laboratory Hubs with a screening approach should accelerate clinical care improvements rapidly. In addition, a regional or national Diagnostic Laboratory Hubs can establish the burden of several diseases and develop rational screening programs according to early results. A well-designed delivery of clinical samples to the hub as well as training and quality control programs could provide global access to diagnosis and decrease the mortality of fungal diseases everywhere as the Guatemala program has shown. Nowadays diagnostic techniques variability is minimal compared with the variability of medical practice. Diagnosis of life-threatening diseases should not rely solely on clinical training but be reinforced by provision of valuable diagnostic information. Implementation of Diagnostic Laboratory Hubs with screening programs would allow improvements in patient care more quickly and with a lower variability than medical training programs alone, for most 
fungal diseases. Laboratory Diagnostic Hubs are also cost-effective means of providing high quality access to diagnosis of fungal diseases.

Author Contributions: Conceptualization, NM, AAI, and JLRT; Data curation and Resources, OB, OG; Writing - original draft, NM, LA, AAI, and JLRT; Writing - review \& editing NM, LRS, AAI, and JLRT, JCP, EA and DWD.

Funding: This work was supported by Global Action Fund for Fungal Infections and JYLAG, a charity Foundation based in Switzerland (E.A. received this funding under the proposal: "Minimising HIV deaths through rapid fungal diagnosis and better care in Guatemala"). Other contributions came from Intrahealth International and the Ministry of health in Guatemala (MSPAS).

Acknowledgments: Fungired Group: (i) Oscar Eduardo López Pérez. Hospital La Amistad JapónGuatemala, Izabal; (ii) Brenan Ortiz Barrientos. Hospital General San Juan de Dios, Guatemala city; (iii) Vilma Alejandrina Reyes Muñoz. Hospital Nacional “Dr Jorge Vides Molina,” Huehuetenango; (iv) Gladys Sajché Aguilar. Hospital Nacional "Juan José Ortega” Coatepeque, Quetzaltenango; (v) Aura Marina Méndez Andrade. Hospital Nacional de Escuintla, Escuintla; (vi) Luis Roberto Santa Marina de León. Hospital Nacional de Malacatán, San Marcos; (vii) Ana Lucía Gómez Alcázar. Hospital Nacional de Occidente, Quetzaltenango; (viii) Eduardo Celada González. Hospital Nacional de Retalhuleu, Retalhuleu; (ix) Gustavo A. Quiñónez M. Hospital Nacional Infantil “Elisa Martínez," Izabal; (x) Germán Orlando Cuyuch Sontay and Marco Paez, Hospital Regional “Hellen Lossi de Laugerud," Alta Verapaz; (xi) Alba Virtud Contreras Marín. Hospital Regional de Cuilapa, Santa Rosa; (xii) María de Lourdes Fong Araujo. Hospital Regional de San Benito, Petén, (xiii) Claudia Mazariegos L. Hospital Regional de Zacapa, Zacapa and (xiv) Brenda Guzmán. Diagnostic Laboratory Hub, Asociación de Salud Integral, Guatemala City.

Conflicts of Interest: A. A-I. has received in the last three years honoraria as a speaker from Gilead Sciences and Pfizer outside the submitted work. E.A. has received honoraria from GILEAD for educational conferences and participation in Advisory board meeting. D. W. D. holds Founder shares in F2G Ltd, a University of Manchester spin-out antifungal discovery company, in Novocyt, which markets the Myconostica real-time molecular assays and has current grant support from the National Institute of Allergy and Infectious Diseases, National Institute of Health Research, North West Lung Centre Charity, Medical Research Council, Global Action Fund for Fungal Infections and the Fungal Infection Trust. He acts or has recently acted as a consultant to Astellas, Sigma Tau, Basilea, Biosergen, Cidara and Pulmocide. In the past 3 years, he has been paid for talks on behalf of Astellas, Dynamiker, Gilead, Merck and Pfizer. He is also a member of the Infectious Disease Society of America Aspergilosis Guidelines and European Society for Clinical Microbiology and Infectious Diseases Aspergillosis Guidelines groups. All other authors declare no conflicts of interest

\section{References}

1. PAHO Diagnosing and Managing Disseminated Histoplasmosis among People Living with HIV; 2020; ISBN 9789275122488.

2. Sifuentes-Osornio, J.; Corzo-León, D.E.; Ponce-De-León, L.A. Epidemiology of Invasive Fungal Infections in Latin America. Current Fungal Infection Reports 2012, 6, 23-34, doi:10.1007/s12281-011-0081-7.

3. Nacher, M.; Blanchet, D.; Bongomin, F.; Chakrabarti, A.; Couppié, P.; Demar, M.; Denning, D.W.; Djossou, F.; Epelboin, L.; Govender, N.; et al. Histoplasma Capsulatum Antigen Detection Tests as an Essential Diagnostic Tool for Patients with Advanced HIV Disease in Low and Middle Income Countries: A Systematic Review of Diagnostic Accuracy Studies. PLOS Neglected Tropical Diseases 2018, 12, e0006802, doi:10.1371/journal.pntd.0006802.

4. Cáceres, DH, Samayoa, BE, NG Medina, AM Tobón, BJ Guzmán, D Mercado, A Restrepo, T Chiller, EE Arathoon, G.B.L. Multicenter Validation in Two Latin American Countries of Commercial Antigen Reagents for the Diagnosis of Histoplasmosis in People Living with HIV/AIDS. Journal of Clinical Microbiology 2018, 56, 1-10, doi:10.1128/JCM.01959-17. 
5. Medina, N.; Alastruey-izquierdo, A.; Mercado, D.; Aguirre, L.; Samayoa, B.; Bonilla, O.; Pérez, J.C.; Rodrigueztudela, J.L. Comparative Performance of the Laboratory Assays Used by a Diagnostic Laboratory Hub for Opportunistic Infections in People Living with HIV. AIDS 2020, doi:10.1097/QAD.0000000000002631.

6. Cáceres, D.H.; Samayoa, B.E.; Medina, N.G.; Tobón, A.M.; Guzmán, B.J.; Mercado, D.; Restrepo, A.; Chiller, T.; Arathoon, E.E.; Gómez, B.L. Multicenter Validation of Commercial Antigenuria Reagents To Diagnose Progressive Disseminated Histoplasmosis in People Living with HIV/AIDS in Two Latin American Countries. Journal of Clinical Microbiology 2018, 56, doi:10.1128/JCM.01959-17.

7. Medina, N.; Alastruey-Izquierdo, A.; Bonilla, O.; Gamboa, O.; Mercado, D.; Pérez, J.C.; Salazar, L.R.; Arathoon, E.; Denning, D.W.; Luis Rodriguez-Tudela, J. A Rapid Screening Program for Histoplasmosis, Tuberculosis, and Cryptococcosis Reduces Mortality in HIV Patients from Guatemala. J. Fungi 2021, 7, 268, doi:10.3390/jof7040268.

8. Samayoa, B.; Aguirre, L.; Bonilla, O.; Medina, N.; Lau-Bonilla, D.; Mercado, D.; Moller, A.; Perez, J.C.; AlastrueyIzquierdo, A.; Arathoon, E.; et al. The Diagnostic Laboratory Hub: A New Health Care System Reveals the Incidence and Mortality of Tuberculosis, Histoplasmosis, and Cryptococcosis of PWH in Guatemala. Open Forum Infectious Diseases 2020, 7 (1), doi:10.1093/ofid/ofz534.

9. Ministerio de Salud Pública y Asistencia Social, G. Guía de Uso de Los Antirretrovirales En Personas Con VIH y Su Aplicación Profiláctica. 2019.

10. Adenis, A.A.; Valdes, A.; Cropet, C.; McCotter, O.Z.; Derado, G.; Couppie, P.; Chiller, T.; Nacher, M. Burden of HIV-Associated Histoplasmosis Compared with Tuberculosis in Latin America: A Modelling Study. The Lancet Infectious Diseases 2018, 18, 1150-1159, doi:10.1016/S1473-3099(18)30354-2.

11. Medina, N.; Samayoa, B.; Lau-Bonilla, D.; Denning, D.W.W.; Herrera, R.; Mercado, D.; Guzmán, B.; Pérez, J.C.C.; Arathoon, E. Burden of Serious Fungal Infections in Guatemala. European Journal of Clinical Microbiology and Infectious Diseases 2017, 36, 965-969, doi:10.1007/s10096-017-2920-0.

12. Adenis, A.A.; Valdes, A.; Cropet, C.; Mccotter, O.Z.; Derado, G.; Couppie, P.; Chiller, T.; Nacher, M. Burden of HIV-Associated Histoplasmosis Compared with Tuberculosis in Latin America : A Modelling Study. The Lancet Infectious Diseases 2018, 3099, 1-10, doi:10.1016/S1473-3099(18)30354-2.

13. Caceres, D.H.; Valdes, A. Histoplasmosis and Tuberculosis Co-Occurrence in People with Advanced HIV. Journal of fungi (Basel, Switzerland) 2019, 73, doi:10.3390/jof5030073.

14. Samayoa, B.; Roy, M.; Cleveland, A.A.; Medina, N.; Lau-Bonilla, D.; Scheel, C.M.; Gomez, B.L.; Chiller, T.; Arathoon, E. High Mortality and Coinfection in a Prospective Cohort of Human Immunodeficiency Virus/Acquired Immune Deficiency Syndrome Patients with Histoplasmosis in Guatemala. The American Journal of Tropical Medicine and Hygiene 2017, 97, 42-48, doi:10.4269/ajtmh.16-0009.

15. Falci, D.R.; Monteiro, A.A.; Braz Caurio, C.F.; Magalhães, T.C.O.; Xavier, M.O.; Basso, R.P.; Melo, M.; Schwarzbold, A. V; Ferreira, P.R.A.; Vidal, J.E.; et al. Histoplasmosis, An Underdiagnosed Disease Affecting People Living With HIV/AIDS in Brazil: Results of a Multicenter Prospective Cohort Study Using Both Classical Mycology Tests and Histoplasma Urine Antigen Detection. Open Forum Infectious Diseases 2019, 6, doi:10.1093/ofid/ofz073.

16. Taylor, R.L.; Dobrovolnyt, A.G.; Dobrovolnyt, charles G.; Dobrovolny, C.G. The Distribution of Histoplasmin Sensitivity in Guatemala. Am J Trop Med Hyg. 1960, Sep, 518-522, doi:DOI: 10.4269/ajtmh.1960.9.518.

17. Programa Nacional de Prevención y Control de ITS VIH y SIDA, P. Informe Nacional de La Cascada Del Continuo de Atención En VIH. 2019, 24, 13-35. 